\title{
About theoretical peculiarities of lowest excitations in modified nanodiamond color centers
}

\author{
A.V.Luzanov \\ SSI "Institute of Single Crystals", STC "Institute for Single Crystals", \\ National Academy of Sciences of Ukraine, \\ 60 Nauky Ave., 61001 Kharkiv, Ukraine
}

Received September 7, 2016

The moderate-size carbon nanoclusters with paramagnetic color centers are studied by using a rather good-working simplified scheme of CNDOL type. Various electronic structure aspects of the clusters are studied. These are the localization of molecular orbitas, the electronic excitation localization and charge-transfer structure of the lowest triplet-triplet transitions, spin density distributions and spin correlations in the ground and excited states. The comparison is made between the respective characteristics of the diamondoid with nitrogen-vacancy $\left(\mathrm{NV}^{-}\right)$and oxygen-vacancy color centers. It is shown that in the asymmetrical $\mathrm{NV}^{-}$center, significant variations of excitation localization and charge/spin transfer take place whereas the energetic properties vary slightly.

Keywords: color center, orbital localization, triplet-triplet transitions, excitation indices, charge transfer, spin density.

C помощью достаточно надежной приближенной схемы типа CNDOL изучены углеродные нанокластеры среднего размера, содержащие парамагнитные центры окраски. Исследованы различные аспекты электронной структуры таких кластеров. К ним относятся локализация МО, локализация электронных возбуждений и зарядово-переносная структура нижайших триплет-триплетных переходов, распределение спиновой плотности и спиновые корреляции в основном и возбужденном состояниях и др. Делается сравнение соответствующих характеристик, вычисленных для диамондоида с азот-вакансионным $\left(\mathrm{NV}^{-}\right)$и с кислород-вакансионным центрами окраски. Показано, что в асимметричном $\mathrm{NV}^{-}$центре имеют место значительные вариации локализации возбуждений и переноса заряда/спина, тогда как энергетические характеристики изменяются слабо.

Стосовно особливостей теоретичного описування найнижчих збуджень у модифікованих центрах забарвлення нанодіамантів. А.В.Лузанов.

Вуглецеві нанокластери середнього розміру, що містять парамагнітні центри забарвлення, вивчено за допомогою доволі надійної наближеної схеми за типом CNDOL. Досліджено різноманітні аспекти електронної будови таких нанокластерів. Це - локалізация МO, локалізація електронних збуджень та зарядово-переносна структура найнижчих триплет-триплетних переходів, розподіл спінової густини та спінові кореляції в основому та збудженому станах тощо. Зроблено порівняння відповідних характеристик діамондоїда з нітроген-вакансійним $\left(\mathrm{NV}^{-}\right)$та оксиген-вакансійним центрами забарвлення. Знайдено, що в асиметричному $\mathrm{NV}^{-}$-центрові має місце значна зміна локалізації збудження та переносу заряду або спіну, тоді як енергетичні властивості змінюються незначною мірою. 


\section{Introduction}

This paper reports some further results from our quantum chemical investigations of excited states in nanodiamonds with paramagnetic color centers. In the earlier papers [1-4] we presented theoretical studies of the electronic structure of such nanostructures, using a simplified CNDO semiempirical technique and the extended Huckel method. Now we perform a more wide analysis while staying within the semiempirical methodology again.

The main distinctions of this work, compared to the above cited papers, are the following. First, a set of systems used in quantum chemical simulation is extended by including structures with high-symmetry color centers and analyzing the typical effects of possible symmetry-lowering. Second, a more refined non ab initio scheme [5-8] is employed here. Third, charge-transfer and spin correlations in the lowest electronic transitions are given in detail. Furthermore, we present a suitable modification of the well-known Lanczos technique [9] (together with its semiempirical implementation) focused on solving large scale eigenvalue problems which inevitably arise in studying the modeled nanoclusters with hundreds atoms.

\section{Computational details}

In the following, we briefly outline the approaches and the subjects of investigation in our modeling of single color centers in nanodiamonds. We apply here a more appropriate CNDO technique than that used before in [1-4]. This is the so-called CNDOL scheme proposed long ago in [5]. Importantly, recent papers $[7,8]$ confirmed a good performance of CNDOL for large carbon-containing networks. Moreover, unlike [1-4], in the present paper we take more regular initial nanodiamond structures, and from these the lower-symmetry color centers can be made in a certain way.

Specifically, the $T_{d}$ symmetry carbon cage having the initial composition $C_{175}$ was generated by a restricted set of translations of diamond-lattice basis vectors (with $d_{\mathrm{C}-\mathrm{C}}$, length of $\mathrm{C}-\mathrm{C}$ bond being taken to $1.54 \AA$ ); the terminal atoms in this $C_{175}$ were saturated by hydrogen atoms. The obtained $T_{d}$ symmetry diamondoid $\mathrm{C}_{175} \mathrm{H}_{116}$ was then routinely optimized by the semiempirical AM1 method (using the Gaussian package [10]). In the resulting configuration of $\mathrm{C}_{175} \mathrm{H}_{116}$ (see the first and second panel in Fig. 1) we made a carbon vacancy located in the geometric center of the diamondoid. Then to produce the NV color center, one carbon atom adjacent to the carbon vacancy was replaced by the nitrogen atom. This leaded to a $C_{3 v^{-s y m m e t r y ~} \mathrm{NV}}$ color center (the typical situation of interest). The vicinity of the NV center is shown in a conditional manner in the third panel of Fig. 1 , where $C_{\alpha}, C_{\beta}$, and $C_{\gamma}$ signify the dangling (unsaturated) carbon atoms surrounding vacancy (V). No additional geometry optimization calculation was performed for the resultant $\mathrm{C}_{173} \mathrm{H}_{116} \mathrm{~N}^{-}$-structure.

Notice that $C_{3 v}$-symmetry color centers, and those of a lower symmetry differ considerably in some important points, and this difference will be a subject of our scrutiny as well. Next, by replacing in the $\mathrm{NV}^{-}$center the nitrogen atom by the oxygen atom we obtain the $C_{3 v}$-symmetry nanocluster $\mathrm{C}_{173} \mathrm{H}_{116} \mathrm{O}$ as a reasonable model for the oxygen vacancy (OV) color centers The latter have received some attention in the last years [11-13]. Furthermore, we consider the lower-symmetry $\mathrm{NV}^{-}$-center in which the vacancy, previously situated in the center of the nanocluster, is shifted to a nearest atom, as in the last panel of Fig. 1. The obtained $C_{s}$ symmetry structure is named here the nanodiamond with shifted NV cen-
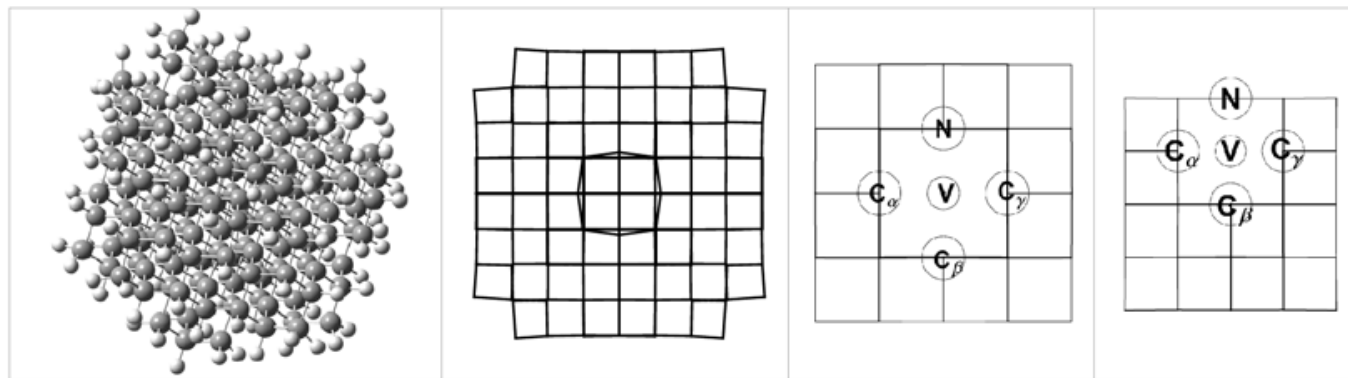

Fig. 1. Spatial image of $\mathrm{C}_{175} \mathrm{H}_{116}$ (the first panel), its plane projection with suppressed hydrogens (the second panel); vicinity of $C_{3 v}$ symmetry $\mathrm{NV}^{-}$center (the third panel), and vicinity of $C_{s}$ symmetry $\mathrm{NV}^{-}$[shift] center. 


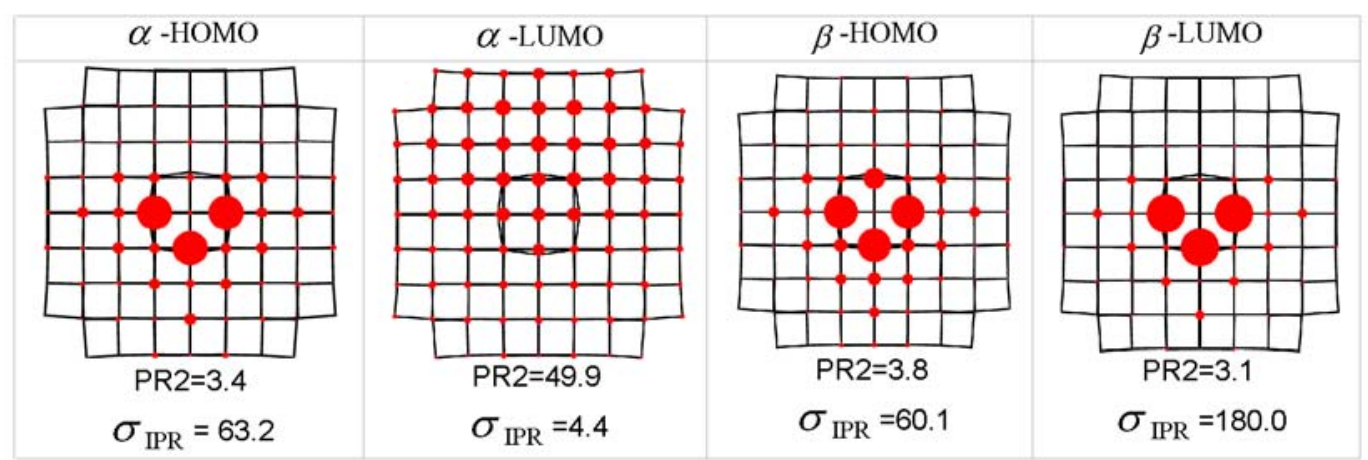

Fig. 2. $C_{3 v}$ symmetry $\mathrm{NV}^{-}$center: localization diagrams for frontier MOs.

ter, or shortly nanodiamond with $\mathrm{NV}^{-}$ [shift] center.

Using such finite-size molecular models, we provide below a detailed CNDOL theoretical study of the defined color centers in nanodiamonds. Namely, we consider properties of the frontier molecular orbitals (MOs), spin behavior of the ground and excited triplet states (spin density and spin correlators), localization and charge transfer in the low-lying triplet-triplet (TT) transitions, and others. We will recall and make more exact the relevant definitions as we go along. The algorithmic features and a modification that we made for UHF (unrestricted Hartree-Fock) computations of excitation spectra within CIS (configuration interaction singles) approach are given in the Appendix. In the latter the results of computations for a larger diamondoid with color center, $\mathrm{C}_{273} \mathrm{H}_{172} \mathrm{~N}^{-}$, are shortly discussed as well.

\section{Orbital properties}

We start with the analysis of MOs because they directly determine many important properties of low-lying electronic states in normal (not too correlated) many-electron systems. Localization properties of frontier MOs, that is HOMOs (highest occupied MOs) and LUMOs (lowest unoccupied MOs), will be the main concern in this section. The nanocluster structures being studied are sufficiently complex, and orbital pictorial images for them are not clear enough to be understood easily. Instead of such fairly intricate spatial images we will display simplified plane projections of the relevant distributions over atoms. In addition, we supply each frontier MO with two quantitative localization measures which are recently studied in [4]. The first localization measure is PR2 index from [4] (Eq. (8) in loc. cit.). It gives an effective number of atomic centers on which the given MO is mostly localized. Another index is $\sigma_{\text {IPR }}$ (Eq. (13) in loc. cit.). This quantity takes a small value of order 1 when MO is delocalized (extended) over the whole system. On the contrary, the $\sigma_{\text {IPR }}$ index is larger by orders of magnitude when the atomic distribution of the MO in question is strongly non-homogeneous and localized one.

More specifically, for the pristine diamondoid $\mathrm{C}_{175} \mathrm{H}_{116}$ we observed a usual (sufficiently delocalized) behavior of the frontier orbitals: PR2 $=40.8$ and $\sigma_{\mathrm{IPR}}=5.3$ for HOMO, and PR2 $=57.7$ and $\sigma_{\text {IPR }}=3.9$ for LUMO of the singlet ground state. The sharply different results are obtained when analyzing the ground triplet state of color centers. For $\mathrm{NV}^{-}$center, HOMOs of $\alpha$ - and $\beta$-shells (related to spin-up and spin-down electrons, respectively) are given in Fig. 1. We see that $\alpha$-HOMO and $\beta$-HOMO, as well as $\beta$-LUMO, are preferably localized in a vicinity of the vacancy, and it is in agreement with the PR2 and $\sigma_{\text {IPR }}$ values given in the same figure. Evidently for the frontier MOs, the most important are three dangling carbon atoms in this defect. However, $\alpha$-LUMO turns out to be strongly delocalized on an upper part of the structure. The given peculiarities of HOMOs and LUMOs are reflected in the locality of electronic transitions considered in the next section.

The data for the corresponding nanodiamond with the OV center mainly show a similar pattern, and we do not display them explicitly. However, in the instant case, unlike the above case, $\alpha$-LUMO is significantly concentrated at the OV color center (almost as for $\alpha$-HOMO in Fig. 1). This feature is consistent with the corresponding values $\mathrm{PR} 2=4.2$ and $\sigma_{\mathrm{IPR}}=56.3$ for the $\alpha$-LUMO. We also mention that in the nanodiamonds having $\mathrm{NV}^{-}$and $\mathrm{OV}$ centers with the $C_{3 v}$ symmetry, $\alpha$-HOMO and $\alpha$-LUMO are doubly degenerate, with symmetry $e$. However, 

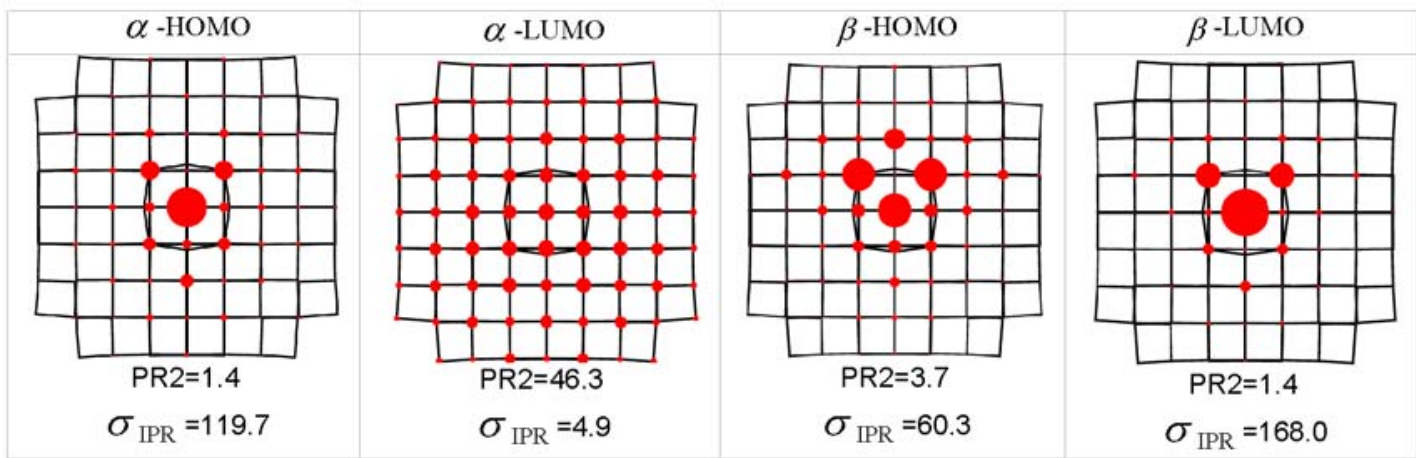

Fig. 3. $C_{s}$ symmetry $\mathrm{NV}^{-}$[shift] center: localization diagrams for frontier MOs.

$\beta$-HOMO is nondegenerate of totally symmetric type $a_{1}$, and $\beta$-LUMO is degenerate of symmetry $e$.

As to nanodiamond with the $C_{s}$-symmetry $\mathrm{NV}^{-}$[shift] center, the situation also seems to be quite similar to that of the $C_{3 v^{\text {-sym- }}}$ metry $\mathrm{NV}^{-}$center. But some peculiarities of this case (see Fig. 3) require remarks. Recall that the vacancy center is now positioned at the fourth line of atomic centers in Fig. 3, just above the geometric center of the cluster. With this, the geometric center (origin site) is now not void, being occupied by a dangling carbon atom. Other two dangling centers are neighbors of this origin site (in Fig. 3 they are most marked, together with the origin). Thus, it is clear from inspecting the images in Fig. 3 that three dangling centers are not fully equivalent in the $\mathrm{NV}^{-}$[shift] color defect, and among them the dangling carbon atom at the origin site is the most active for all frontier MOs. Notice that the $\alpha$-HOMO is significantly delocalized, as in the normal $\mathrm{NV}^{-}$center. Remark also that in most images the distal (background) atoms are superimposed over the vacancy center.

We very shortly discuss the frontier orbital energies $\varepsilon$. Unlike the correctly constructed spin-restricted open-shell MOs, the canonical UHF orbitals cannot be interpreted as Koopmans orbitals [14]. That is why we give only the HOMO-LUMO orbital gap which can serve as a crude estimation of the fundamental gap $\Delta \varepsilon$ in solid state physics (the difference between ionization energy and electron affinity) [15]. This quantity is usually symbatic with the lowest excitation energy.

Within the UHF approximation we make a distinction between $\Delta \varepsilon$ for $\alpha$-shell $\left(\Delta \varepsilon^{\alpha}\right)$ and $\Delta \varepsilon$ for $\beta$-shell $\left(\Delta \varepsilon^{\beta}\right)$. For instance, $\Delta \varepsilon^{\alpha}=$ $\varepsilon_{\text {LUMO }}{ }^{\alpha}-\varepsilon_{\text {HOMO }}{ }^{\alpha}$. In the adopted CNDOL model the following values (in eV) are ob- tained for the $\mathrm{NV}^{-}, \mathrm{OV}$ and $\mathrm{NV}^{-}$[shift] centers, respectively:

$$
\begin{gathered}
\Delta \varepsilon^{\alpha}=\{9.78,9.25,9.70, \\
\Delta \varepsilon^{\beta}=\{6.70,6.91,6.70
\end{gathered}
$$

We return to them in the next Section, and now only remark that $\Delta \varepsilon^{\beta}$ is notably lower than $\Delta \varepsilon^{\alpha}$. Thus, we can expect that the participation of spin-down electrons must be the most important when forming the lowest (TT) transitions.

\section{Localization and other properties of TT transitions}

The interpretation of configuration interaction wave functions in terms of local and charge-transfer electronic transitions has a rather long history, starting with the work of Longuet-Higgins [16]. Seemingly, the appropriate quantitative indices were first introduced in [17-19], and now there are a variety of related indices for a similar analysis of sophisticated electronic excitations (for recent advances see [2, 20-24]). We follow our previously given technique of excitation indices and charge transfer numbers [18, 19]; the technique is also named the excited state structural analysis (ESSA) [20]. Preliminary results of applying ESSA to $\mathrm{NV}$ centers are described in [2]. We report here a more extended study of the analogous model systems.

For convenience we recall the main points of ESSA for the CIS model. Two sets of atomic-centered quantities are introduced into the consideration. These are excitation localization indices $\left\{L^{*}{ }_{A}\right\}$ and charge transfer (CT) numbers $\left\{l_{A \rightarrow B}\right\}$. For a given atom $A$, excitation index $L_{A}^{*}$ determines a measure of participation of the atom in the excitation considered. Further, for the given atom pair $(A, B)$, CT number $l_{A \rightarrow B}$ determines a probability of appearing the se- 
Table 1. The calculated properties of ${ }^{3} A_{1} \rightarrow{ }^{3} E$ transition in $\mathrm{NV}^{-}$and $\mathrm{OV}$ color centers generated in pristine nanodiamond $\mathrm{C}_{175} \mathrm{H}_{116} \cdot \lambda$ is transition energy in eV (oscillator strength $f$ in parentheses); $\left\|\tau_{\beta}\right\|^{2}$ is the contribution of spin-down one-electron transitions to the total CIS excitation, and $\left\langle\boldsymbol{S}^{2}\right\rangle^{*}$ its squared total spin; $\kappa_{\mathrm{CIS}}$ is the collectivity number, and PR2* is the PR2 localization index for the transition

\begin{tabular}{|c|c|c||}
\hline & $\mathrm{NV}^{-}$center & OV center \\
\hline$\lambda(f)$ & $1.960(0.064)$ & $2.183(0.077)$ \\
$\left\|\tau_{\beta}\right\|^{2}$ & 0.981 & 0.979 \\
$\left\langle S^{2}\right\rangle^{*}$ & 2.008 & 2.004 \\
$\kappa_{C I S}$ & 1.088 & 1.098 \\
$\mathrm{PR} 2^{*}$ & 3.15 & 3.12 \\
\hline
\end{tabular}

lected "ion structure" $\left|\ldots A^{+} \ldots B^{-} \ldots\right\rangle$ in the excited state (implying that the ground state symbolically corresponds to $|. . A . . B . .\rangle$.$) .$ In $[2,3,20]$, working expressions are given for these indices in terms of the CIS coefficients (particle-hole amplitudes) and MOs; see also Eq. (16) in the Appendix.

Turning to our problems, we first consider the $C_{3 \mathrm{v}}$ symmetry color centers. Table 1 presents the computed properties of the lowest TT transition in NV and OV centers. Worth noting is the fact that, judging from Table 1, spin-down electrons give the predominant contribution to the TT transition (the squared norm of the excited spinup electrons is $\left\|\tau_{\alpha}\right\|^{2} \equiv 1-\left\|\tau_{\beta}\right\|^{2}$ ). Furthermore, collectivity number $\kappa_{\text {CIS }}[19,20]$ tells us that the transition is almost oneconfigurational one. Therefore, the $\beta$-frontier MOs studied in the preceding section, quite predetermine the nature of the lowest transition. That this transition is strongly localized follows from the PR2* values given in the table. Below this peculiarity will be considered with more scrutiny.

The corresponding images presenting distributions of excitation indices $\left\{L_{A}^{*}\right\}$ and $\left\{\Delta q_{A}^{*}\right\}$ (charge density differences) for the lowest ${ }^{3} E$ excitation are given in Fig. 4 . We observe that this excitation is indeed significantly (more than $70 \%$ ) localized in the vicinity of four atoms forming the $\mathrm{NV}^{-}$center. If extending the region of interest by a subcluster of radius $3 \AA$ (it contains $\mathrm{N}$-atom, three dangling atoms and, in addition, 29 carbon atoms), then we find that only $85 \%$ of the excitation will be captured even in this case. In other words, even distal atoms fairly

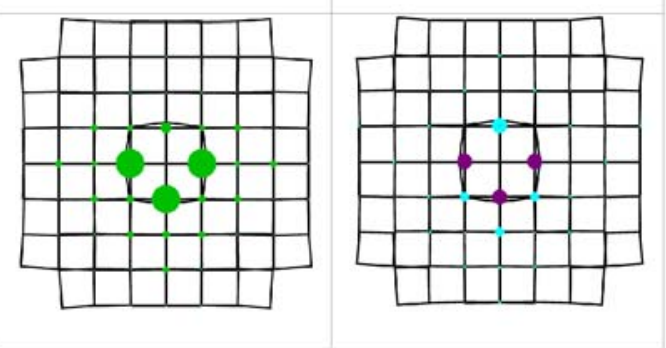

Fig. 4. Images for excitation indices (green) and charge variations for the lowest ${ }^{3} E$ excited state in $C_{3 v}$ symmetry $\mathrm{NV}^{-}$center. The positive charge variations are in dark magenta, and negative ones in cyan.

markedly contribute to the resulting excitation. The same features are characteristic of the OV center. Notice also that replacement of the nitrogen by the oxygen (a more electronegative atom) causes a hypsochromic shift about $0.2 \mathrm{eV}$ (see Table 1) which is in an accordance with changing $\Delta \varepsilon^{\beta}$ in Eq. (1).

The above mentioned CT numbers additionally clarify the nature of the ${ }^{3} E$ state. There are two "global" CT characteristics [20]. The first is a total measure of the CT character:

$$
\mathrm{CT}_{\text {tot }}=1-\sum_{A} l_{A \rightarrow A}
$$

The second is the excitation radius

$$
R_{e x c}=\sum_{A, B} l_{A \rightarrow B}\left|\boldsymbol{R}_{A}-\boldsymbol{R}_{\boldsymbol{B}}\right|
$$

where $\mathbf{R}_{A}$ stands for the Cartesian coordinate vector of atom $A$. In the case of the ${ }^{3} E$ state considered, $\mathrm{CT}_{\text {tot }}=82 \%$, that is, the excitation is predominantly of CT character. With this, the local (near the vacancy vicinity) interatomic CT processes are significant, so that $R_{\text {exc }}=2.652 \AA$ (compare it with $\sqrt{8 / 3} d_{\mathrm{C}-\mathrm{C}}=2.515 \AA$, the distance between dangling carbon atoms $C_{\alpha}$ and $C_{\beta}$ in the perfect lattice). For completeness we present the matrix of CT numbers for all vacancy vicinity atoms $\mathrm{N}, \mathrm{C}_{\alpha}, \mathrm{C}_{\beta}$, and $\mathrm{C}_{\gamma}$ :

$$
\left\|l_{A \rightarrow B}\right\|=|| \begin{array}{llll}
0 . & 0.017 & 0.017 & 0.017 \\
0.0 .052 & 0.050 & 0.050 \\
0 . & 0.050 & 0.052 & 0.050 \\
0.0 .050 & 0.050 & 0.052
\end{array} \mid .
$$

Generally, CT matrix is nonsymmetric, but in the case we have $l_{A \rightarrow B}=l_{B \rightarrow A}$ for three equivalent sites $C_{\alpha}, C_{\beta}$ and $C_{\gamma}$. As a result, $\Delta q^{*}$ (electronic charge variation due 


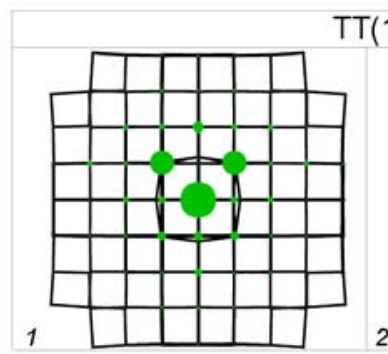

TT(1)

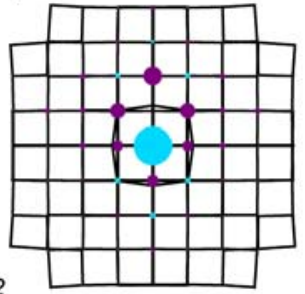

$\mathrm{TT}(2)$

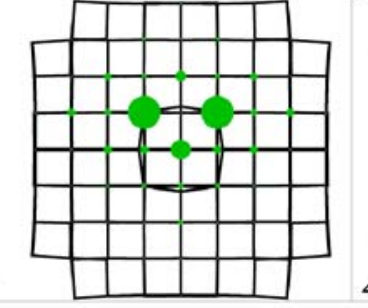

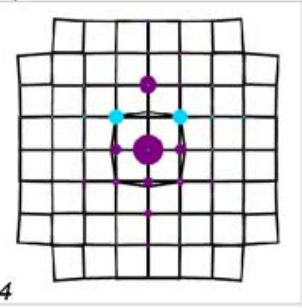

Fig. 5. Excitation indices $(1,3)$ and charge variation diagrams for the $C_{s}$ symmetry $\mathrm{NV}^{-}$[shift] center for the two lowest transitions $\mathrm{TT}(1)$ and $\mathrm{TT}(2)$. The positive charge variations are in dark magenta, and negative ones in cyan.

to excitation) is rather small: $\Delta q^{*}{ }_{\mathrm{N}}=0.065$, $\Delta q^{*} \mathrm{C}_{\alpha}=\Delta q^{*} \mathrm{C}_{\beta}=\Delta q^{*} \mathrm{C}_{\gamma}=-0.061$.

Somewhat different picture is observed for the $\mathrm{NV}^{-}$[shift] center. The corresponding visual diagrams for excitation indexes and charge variations are exhibited in Fig. 5. In this case, the degenerate term ${ }^{3} E$ is spit due to symmetry-lowering from $C_{3 v}$ to $C_{s}$, and now we have two quasidegenerate triplet excitations, $T_{(1)}$ and $T_{(2)}$, with transition energies $\lambda_{(1)}=1.894 \mathrm{eV}$ and $\lambda_{(2)}=$ $2.088 \mathrm{eV}$ (the average oscillator strength $f$ is about the previously given value in Table 1 , namely $f=0.061$ ). In the diagrams we see that dangling atom $\mathrm{C}_{\beta}$ (which is situated in the geometric origin of the structure) is the most active in excitation $T_{(1)}$ whereas two other dangling atoms $C_{\alpha}$ and $C_{\gamma}$ are most active in $T_{(2)}$. This behavior is in accordance with the localization of frontier spin-down MOs which are displayed in Fig. 3. The following CT matrices of type (4),

$$
\begin{aligned}
& \left\|l_{A \rightarrow B}\right\|{ }^{\left[T_{(1)}\right]}=|| \begin{array}{llll}
0 . & 0.035 & 0.010 & 0.010 \\
0 . & 0.110 & 0.030 & 0.030 \\
0 . & 0.093 & 0.028 & 0.026 \\
0 . & 0.093 & 0.026 & 0.028
\end{array}|| ; \\
& \left.\left\|l_{A \rightarrow B}\right\| \|_{(2)}\right]=|| \begin{array}{llll}
0 & 0.000 & 0.028 & 0.028 \\
0 . & 0.000 & 0.082 & 0.082 \\
0 . & 0.000 & 0.077 & 0.074 \\
0 . & 0.000 & 0.074 & 0.077
\end{array}||
\end{aligned}
$$

show a definite difference in the CT structure of $T_{(1)}$ and $T_{(2)}$ in the vacancy vicinity. As a result, the electronic charge on $\mathrm{C}_{\beta}$ is decreased in $T_{(1)}\left(\Delta q^{*} \mathrm{C}_{\beta}=-0.277\right)$, and increased in $T_{(2)}\left(\Delta q^{*} \mathrm{C}_{\beta}=0.203\right)$. These values can be compared with the above given $\Delta q^{*} \mathrm{C}_{\beta}=-0.061$ for ${ }^{3} E$. Therefore, the degeneracy removal in ${ }^{3} E$ comes to a significant charge redistribution in the vacancy

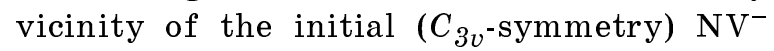
center. The above results for the $\mathrm{NV}^{-}$ [shift] center are in a qualitative agreement with those reported in [2] for a smaller asymmeric cluster examined by a somewhat different approach.

\section{Spin densities and spin correlators}

Spin properties of the single defects being studied are also important because of the triplet nature of the ground state and lowest allowed excitations in many color centers. Here we will discuss the calculated spin characteristics for our main systems. In the case of $\mathrm{C}_{173} \mathrm{H}_{116} \mathrm{~N}^{-}$, distributions of atomic spin densities, $\left\{Q_{A}\right\}$, i.e. differences between local populations of spin-up and spin-down electrons, are portrayed in Fig. 6 where corresponding PR2 indexes are given too. In this figure, we display spin distribution for the ground triplet state and the spin density variation, $\left\{\Delta Q_{A}\right\}$, due to transition ${ }^{3} A_{2} \rightarrow{ }^{3} E$. Explicitly, $\Delta Q_{A} \equiv Q_{A}$ exc $-Q_{A}$, where $Q_{A}$ exc is a spin density on atom $A$ in the excited state considered.

We observe that distribution $\left\{Q_{A}\right\}$ very much resembles $\alpha$-HOMO in Fig. 2. This is not so surprising if we take into account that even within UHF for triplet states, the spin density matrix is largely formed by two unpaired electrons which occupy $\alpha$-HOMO and $\alpha$-(HOMO-1). In our case these orbitals just correspond to the previously studied doubly degenerate HOMO (see the first panel in Fig. 2). The same is true for the other color centers treated here; the detailed data (variations are few percents only) are omitted to conserve space. In both ground and lowest excited states of all the systems, the spin resides basically on the three dangling carbon atoms for (nearly $3 / 4$ of the total spin of norm 2). For example, in the $\mathrm{NV}^{-}$center $Q\left[\mathrm{C}_{\alpha}\right]=0.548$ (in the ground state ${ }^{3} A_{2}$ ) and $Q^{*}\left[C_{\alpha}\right]=0.485$ (in 


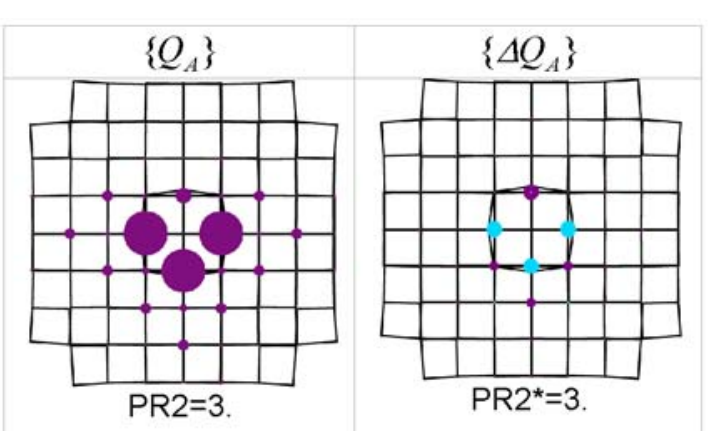

Fig. 6. Spin density distribution $\left\{Q_{A}\right\}$ in the ground state (the left panel) and difference spin density distribution $\left\{\Delta Q_{A}\right\}$ for the lowest ${ }^{3} E$ excited state. The positive spin density differences are in dark magenta, and negative ones in cyan.

the excited state ${ }^{3} E$ ). This small variation of spin densities under excitation (see also the right panel in Fig. 7) is in conformity with the local properties of frontier MOs. For the ground state, a strong spin localization in the vicinity of $\mathrm{NV}$ centers is well established [1, 25-27]. Notice that the experimental situation for the lowest excited state is not so clear.

Further information is provided by calculations of the atomic spin correlators which were introduced in quantum chemistry very long ago [28]. By definition, spin correlator $K_{A B}$ for atomic centers $A$ and $B$ is a quantum mechanical average of the scalar product of local spin operators $\mathbf{S}_{A}$ and $\mathbf{S}_{B}$ for atoms $A$ and $B$, respectively, viz., $K_{A B}=\left\langle\mathbf{S}_{A} \cdot \mathbf{S}_{B}\right\rangle$. For their properties, applications, and computational schemes see, e.g., [29-31]. We present here our semiempirical results for the correlation matrix

$$
K=\left\|K_{A B}\right\|,
$$

containing spin correlators of atoms in the vicinity of $\mathrm{NV}^{-}$center. For the ground ${ }^{3} A_{2}$ and excited ${ }^{3} E$ electronic states the results are

$$
K\left[{ }^{3} A_{2}\right]=|| \begin{array}{cccc}
1.133 & -0.001 & -0.001 & -0.001 \\
-0.001 & 1.532 & 0.053 & 0.053
\end{array}|| \text {; }
$$

where the first row contains the autocorrelation of the $\mathrm{N}$ atom and the correlations of this atom with the nearest dangling atoms $\mathrm{C}_{\alpha}, \mathrm{C}_{\beta}$, and $\mathrm{C}_{\gamma}$; the second row is related to $\mathrm{C}_{\alpha}$, that is one of the equivalent dangling atoms. In these $K$ matrices, we omitted equivalent rows corresponding to other dangling carbon atoms. As seen from Eq. (5),

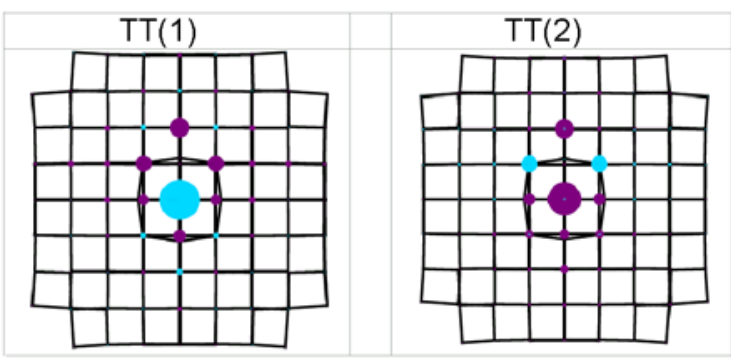

Fig. 7. Spin density distribution $\left\{Q_{A}\right\}$ in the ground state (the left panel) and difference spin density distribution $\left\{\Delta Q_{A}\right\}$ for the two lowest TT transitions in the $C_{s}$ symmetry $\mathrm{NV}^{-}$[shift] center. The positive spin density differences are in dark magenta, and negative ones in cyan.

similar to the spin density distributions, little changes are observed in $K$ due to the electronic transition. We note also that for usual molecular species, spin correlators can serve as a good indicator of valence bondings (correlators are significantly negative for strong chemical interactions). Turning to the above results we find too small spin correlations between the dangling atoms (near 0.05). Interestingly, in the case of $\mathrm{NV}^{-}$[shift] center, even these small correlations almost disappear (near 0.0001). Of course, the spin correlations of valence type are always nonnull; e.g., in $\mathrm{NV}^{-}$center we find $K_{A B}\left[{ }^{3} A_{2}\right]=-0.367$ and $K_{A B}\left[{ }^{3} E\right]=-$ 0.369 for atomic pair $(\mathrm{A}, \mathrm{B})=\left(\mathrm{C} \alpha, \mathrm{C}^{\prime}\right)$ consisted of dangling atom $\mathrm{C}_{\alpha}$ and its chemically bonded nearest neighbor atom $C^{\prime}$.

\section{Conclusions}

In this paper we considered semiempirically the modeled nanodiamonds containing the paramagnetic color centers. Sensible results were obtained by using the CNDOL approach [5] to the study of these modeled systems even with a simplified molecular geometry. In particular, at the adopted level of theory we have got quite satisfactory results for the lowest allowed TT transitions. This allowed us to consider the resulting wave functions as fairly reasonable. On this reason we could safely apply to these wavefunctions the previously given technique [17-19, 20] of the CIS and CISlike structural analysis.

Let us summarize the main results. First, only the spin-down frontier MOs are all strongly localized at the color center vicinity (that is on the heteroatom and dan- 
gling carbon atoms). Second, about $70 \%$ of the lowest TT transition is concentrated in the color center vicinity, so the contribution of the environment into the excitation is not negligible. The lowest excitations have a significant charge transfer character $(80 \%$ or so), and the corresponding charge transfer structure is mostly formed by atoms of the color center vicinity together with atoms of the environment (in a minor extent). Third, atomic spin densities of the ground and excited triplet states are distributed in the same manner as the frontier MOs are localized on atoms. At last, the spin-correlator analysis reveals no significant correlations of electron spins of the dangling atoms.

Now we shortly discuss possible extensions and improvements of the applied techniques. With powerful computers, our approach can be advanced further, making it more available for the treatment of nanoclusters with many hundreds and more carbon atoms. At the same time, the technique applied here is based on solving approximately a many-electron problem for the sufficiently large cluster (see an example in the Appendix). However, this approach precludes us from studying more complex problems. It is worthwhile mentioning that defects are frequently treated theoretically in the framework of the so-called embedded cluster model [32, 33]. Even bulks and huge clusters can be described by various models of this type [34, 35]. The usual strategy in these studies is to construct a classical environment in the form of very large external cluster (e.g., a vast set of static charges) into which a fairly small "quantum mechanical" cluster is immersed. A related approach is the standard combined quantum mechanics and molecular mechanics (QM/MM) approach [36]. Such techniques can be easily reformulated at the semiempirical level, and this opens up an attractive vista of research for the investigators interested in studying excited states of very large-scale nanodiamonds with defects.

Acknowledgement. The work was partially supported by the joint project of the National Academy of Sciences of Ukraine and the National Academy of Sciences of Belarus (Grant No. 09-06-15). The author is grateful to A.P. Nizovtsev for very valuable discussions on the subject of the modified nanodiamond color centers.

\section{Appendix: A modification of the large-scale eigenvalue algorithm for excitation energies at the CIS/UHF level}

Here we consider how to compute in the economical way a lower part of excitation energy spectrum within the full CIS/UHF approximation. Let us first provide the needed formal background. We follow in essence the matrix, more exactly, matrix-covariant procedure described in [37] (its precursors were previously given in works [38, 39]). In the matrix formulation of CIS/UHF theory the key quantities are spinless components $\tau_{\alpha}$ and $\tau_{\beta}$ of the full spin-orbital transition density matrix $\tau$ related to the excited state under study. Matrix elements of $\tau_{\alpha}$ in a MO basis determine particle-hole amplitudes, or simply, excitation amplitudes of spin-up electrons; the $\tau_{\beta}$ matrix elements do that for spin-down electrons. Under this construction, the optimal transition density matrix $\tau$ is but an eigenvector of the corresponding excitation Hamiltonian matrix $\Pi$, so

$$
\Pi(\tau)=\lambda^{*} \tau,
$$

with eigenvalue $\lambda^{*}$ being the respective transition energy. The more detailed is the following coupled set of equations (Eqs. (56) and (57) in [37]):

$$
\Pi\left(\tau_{\alpha}, \tau_{\beta}\right)=\lambda^{*} \tau_{\alpha}, \quad \Pi\left(\tau_{\beta}, \tau_{\alpha}\right)=\lambda^{*} \tau_{\beta},
$$

where we define an action of $\Pi$ on two arbitrary transition matrices $X_{\alpha}, X_{\beta}$ as follows:

$$
\begin{gathered}
\Pi\left(X_{\alpha}, X_{\beta}\right)= \\
=\left(1-\rho_{\alpha}\right)\left[f_{\alpha} X_{\alpha}-X_{\alpha} f_{\alpha}+J\left(X_{\alpha}+X_{\beta}\right)-K\left(X_{\alpha}\right)\right] \rho_{\alpha},
\end{gathered}
$$

and $\Pi\left(X_{\beta}, X_{\alpha}\right)$ is computed likewise, but with a simultaneous exchange in Eq. (8) all $\alpha$-matrices by $\beta$-matrices and vice versa. Here the conventional notation is used: $f_{\alpha}$ and $f_{\beta}$ are the Fock matrices for spin-up and spin-down electrons; $\rho_{\alpha}$ and $\rho_{\beta}$ are projectors onto corresponding occupied orbitals; and symbols $J$ and $K$ denote, respectively, the Coulomb and exchange operators acting on matrix argument by the standard rules defined by Roothaan. The usual normalization condition to 1 is used for the CIS/UHF transition matrices:

$$
\operatorname{Tr}\left[\left(\tau_{\alpha}\right)^{+}\left(\tau_{\alpha}\right)+\left(\tau_{\beta}\right)^{+}\left(\tau_{\beta}\right)\right]=1
$$


The advantage of the covariant matrix formulation $(7,8)$ is that in calculations we can use any appropriate orbital basis. In particular, for semiempirical schemes the most economical is working with matrices in AO representation. This requires no further four-index matrix operations which appear in the MO formulation of the related so-called direct-CI method. Given the AO basis set of size dim, the generic computation of $\Pi(\tau)$ can be easily implemented, needing only $\approx \mathrm{dim}^{3}$ floating point operations.

For our problem (6) we first outline the Lanczos method which is one of the most efficient technique for solving large-scale incomplete eigenvalue problems (see, e. g., $[40,41])$. In the case of Eq. (6) we start with an initial (usually random) transition matrix $\tau^{[1]}$ of norm unity. Then we make the corresponding recurrence $(1 \leq j \leq k)$ by which the Lanczos vectors $\left\{X^{[j]}\right\}_{1 \leq j \leq k}$ (the Lanczos transition matrices in our case) are built up:

$$
\begin{gathered}
X^{[1]}=\tau^{[1]} \\
\tilde{X}^{[j+1]}=\Pi\left(X^{[j])}-\alpha^{[j]} X^{[j]}-\beta^{[j-1]} X^{[j-1]},(11\right.
\end{gathered}
$$

where $\alpha^{[j]}=\operatorname{Tr}\left(X^{[j]}\right)^{+} \Pi\left(X^{[j]}\right)$, and $\beta^{[j-1]}=$ $\left[\operatorname{Tr}\left(X^{[j-1]}\right)^{+}\left(X^{[j-1]}\right)\right]^{1 / 2} ; \quad$ moreover, $\quad X^{[0]}=0$ and $\beta^{[0]}=0$. From numbers $\alpha^{[j]}$ and $\beta^{[j]}$ the tridiagonal Jacobi matrix $\Pi^{[k]}$ is shaped. Its $j$ th row is of the form:

$$
\pi^{[j]}=\left\|0, \ldots 0, \beta^{[j-1]}, \alpha^{[j]}, \beta^{[j]}, 0, \ldots 0\right\|,
$$

so

$$
\Pi_{[k]}=\|\pi[j]\|_{1 \leq j \leq k}
$$

As a rule, the lowest eigenvector of this matrix ("excitation matrix") provides a fairly good estimation of the first excited state even for rather small sub-dimension $k$. However, frequently we need several goodquality excited states, whereas there is not enough computer memory to store many matrices $\left\{X^{[j]}\right\}_{1 \leq j \leq k}$ when $k$ is not small. Various modifications of the so-called restart Lanczos method could help to obviate this difficulty [42]. And yet, in our case (in fact, we use a laptop for calculations) there is not much memory in volatility, and we are forced to make a simplification of the restart technique.

Our modification is as follows. At the first stage, we perform the Lanczos loop, Eqs. (9)-(12), taking in practice $k=10 \div 20$ (more often than not, we put $k=15$ ). Addi- tionally, we include reorthogonalization operations to correct numerical results of the above Lanczos loop, as described in [40], sect. 13.7. Next, we fully solve the small eigenvalue problem for $\Pi^{[k]}\left(k<<\mid \operatorname{dim}^{4}\right)$. However, to obtain more accurate results we must, at this stage, repeat same process (9)-(12), each time with a new $X^{[1]}$ (not more than ten times, as usual). At each such Lanczos's reiteration the new $X^{[1]}$ is taken as the lowest eigenvector produced by the current excitation matrix $\Pi^{[k]}$. With relatively small $k$, the procedure guarantees quite satisfactory results only for the lowest excitation. Therefore, to compute more excitations we proceed to a more complicated process. Namely, in the second stage we take from the first stage the two lowestenergy solutions, $\tau_{(1)}$ and $\tau_{(2)}$. This matrix $\tau_{(1)}$ is almost good enough for a genuine lowest excitation, whereas matrix $\tau_{(2)}$ is taken as the new start $X^{[1]}$ in the subsequent Lanczos loop (9)-(12). In doing so, we must maintain the orthogonality of all current Lanczos vectors $\left\{X^{[j]}\right\}_{1 \leq j \leq k}$ to matrix $\tau_{(1)}$. As a final result of the second stage, we obtain updated matrix $\tau_{(1)}$ which is now appropriate to the second excitation. The corresponding $\tau_{(2)}$ matrix is used as a start for the next (third) stage and so on. A number $r$ of the stages (outer loops) is determined by a number of the excited states we want to calculate. After completing all $r$ outer loops, we have at our disposal a set of good-quality solutions $\left\{\tau_{i}\right\}_{1 \leq j \leq r}$. Additionally, we control and partly improve the results by computing the overlap matrix

$$
\left\|\operatorname{Tr}\left(\tau_{(i)}\right)^{+} \tau_{(j)}\right\|_{1 \leq i, j \leq r}
$$

(checking orthogonality) and doing the associated generalized eigenvalue problem for the reduced excitation Hamiltonian matrix

$$
\left\|\operatorname{Tr}\left(\tau_{(i)}\right)^{+} \Pi\left(\tau_{(j)}\right)\right\|_{1 \leq i, j \leq r}
$$

In our modified Lanczos-type algorithm, the final eigensolutions $\left\{\tau_{i}\right\}_{1 \leq i \leq r}$ thus obtained correspond as much as possible to the $r$ lowest excited states. The accuracy is normally estimated evaluating the energy variance $\sigma^{2}$ (the residual squared norm) for each excited state. For our purposes $\sigma^{2}=10^{-10}$ atomic units is adopted as an indicator of the good numerical accuracy. For the given $i$ th excitation we have explicitly spin-up and spin-down components $\tau_{(i)}{ }^{\alpha}$ and $\tau_{(i)}{ }^{\beta}$ of $\tau_{(i)}$. Given these components, we compute 
the key CT numbers (within CIS//UHF) as follows:

$$
l_{A \rightarrow B}=\sum_{\mu \in A} \sum_{v \in B}\left(\left|\left\langle\chi_{v}\left|\tau_{(i)}^{\alpha}\right| \chi_{\mu}\right\rangle\right|^{2}+\left|\left\langle\chi_{v}\left|\tau_{(i)}^{\beta}\right| \chi_{\mu}\right\rangle\right|^{2}\right) .
$$

Owing to Eq. (9) they are normalized to unity, as it must be for the probability quantities.

We employed the presented algorithm for all examples given in the paper. Furthermore, we used it to understand how increasing size of the cluster will affect the properties of clusters with single color center. For this we took the enlarged regular diamondoid $\mathrm{C}_{275} \mathrm{H}_{172}$ and built up the $C_{3 v}$ symmetry $\mathrm{NV}^{-}$center of composition $\mathrm{C}_{273} \mathrm{H}_{172} \mathrm{~N}^{-}$. We founded that the change in most electronic properties of this color center is less than percent, as compared to $\mathrm{C}_{173} \mathrm{H}_{116} \mathrm{~N}^{-}$. For instance, spin-down orbital gap $\Delta \varepsilon \beta$ is found to be slightly shifted towards a lower energy: $\Delta \varepsilon^{\beta}=6.671$ (see Eq. (1) for comparison). This shift is consistent with the calculated (bathochromic) shift of ${ }^{3} A_{2} \rightarrow{ }^{3} E$ transition energy: $\lambda=1.946 \mathrm{eV}$ (compare it with the $\lambda$ value from Table 1 ). Notice that in this example we in fact solved implicitly the incomplete eigenvalue problem for $806448 \times 896448$ matrix of the configuration interaction method.

\section{References}

1. A.V.Luzanov, Functional Materials, 22, 514 (2015).

2. A.V.Luzanov, O.A.Zhikol, Functional Materials, 23, 63 (2016).

3. A.V.Luzanov, O.A.Zhikol, I.V.Omelchenko et al., Functional Materials, 23, 268 (2016).

4. A.V.Luzanov, Functional Materials, in press. 23, 599 (2016).

5. L.A.Montero, L.Alfonso, J.R.Alvarez, E.Perez, Int.J.Quantum.Chem, 37, 465 (1990).

6. L.A.Montero-Cabrera, U.Rohrig, J.A.PadronGarcia et al., J.Chem.Phys., 127, 145102 (2007).

7. A.L.Montero-Alejo, M.E.Fuentes, E.Menendez-Proupin et al., Phys.Rev.B, 81, 235409 (2010).

8. A.L.Montero-Alejo, E.Menendez-Proupin, M.E.Fuentes et al., Phys.Chem.Chem.Phys., 14, 13058 (2012).

9. L.Komzsik, The Lanczos Method: Evolution and Application, SIAM, Philadelphia (2003).

10. M.J.Frisch, G.W.Trucks, J.B.Schlegel et al., Gaussian 09, Revision C.01; Gaussian, Inc.: Wallingford, CT, USA (2010).

11. J.P.Goss, P.R.Briddon, M.J.Rayson et al., Phys. Rev. B, 72, 035214 (2005).
12. Y.G.Zhang, Z.Tang, X.G.Zhao et al., Appl. Phys. Lett., 105, 052107 (2014).

13. A.L.Pushkarchuk, S.A.Kuten, V.A.Pushkarchuk et al., in: Proc. XIV Int. Conf. on Quantum Optics and Quantum Information, Minsk, Belarus (2015). p.48.

14. B.N.Plakhutin, E.R.Davidson, J.Chem.Phys., 132, 184110 (2010); E.R.Davidson, AIP Conf. Proc., 1504, 5 (2012).

15. E.J.Baerends, O.V.Gritsenko, R.van Meer, Phys. Chem. Chem. Phys., 15, 16408 (2013).

16. H.C.Longuet-Higgins, J.N.Murrell, Proc. Phys. Soc., A68, 901, 969 (1955).

17. A.V.Luzanov, A.A.Sukhorukov, V.E.Umanski, Theor. Experim. Chem., 10, 354 (1974).

18. A.V.Luzanov, V.F.Pedash, Theor. Experim. Chem., 15, 338 (1979).

19. A.V.Luzanov, Russ.Chem.Rev., 49, 1033 (1980).

20. A.V.Luzanov, O.A.Zhikol, in: Practical Aspects of Computational Chemistry I: An Overview of the Last Two Decades and Current Trends, ed. by J.Leszczynski, M.K.Shukla, Springer, New York (2012), p.415.

21. S.A.Bappler, F.Plasser, M.Wormit, A.Dreuw, Phys. Rev., A90, 052521 (2014).

22. L.Blancafort, A.A.Voityuk, J.Chem.Phys., 140, 095102 (2014).

23. T.Etienne, X.Assfeld, A.Monari, J.Chem.Theory Comput., 10, 3896 (2014); T.Etienne, J. Chem. Phys., 142, 244103 (2015).

24. A.V.Luzanov, D.Casanova, X.Feng, A.I.Krylov, J.Chem. Phys., 142, 224104 (2015).

25. J.Wrachtrup, S.Y.Kilin, A.P.Nizovtsev, Opt. Spectrosc. 91, 429 (2001); A.P.Nizovtsev, S.Ya.Kilin, A.L.Pushkarchuk et al., New J.Phys., 16, 083014 (2014).

26. A.Gali, M.Fyta, E.Kaxiras, Phys.Rev.B, 77, 155206 (2008).

27. I.Kratochvilova, A.Kovalenko, A.Taylor et al., Phys.Stat. Sol. (a), 207, 2045 (2010).

28. W.G.Penney, Proc.Roy.Soc.A, 158, 306 (1937).

29. A.V.Luzanov, Y.F.Pedash, S.Mohamad, Theor. Experim. Chem. 29, 485 (1989); A.V.Luzanov, O.V.Prezhdo, Mol. Phys., 105, 2879 (2007).

30. A.E.Clark, E.R.Davidson, J.Chem.Phys., 115, 7382 (2001); A.E.Clark, E.R.Davidson, Mol. Phys, 100, 373 (2002).

31. A.V.Luzanov, in: Practical Aspects of Computational Chemistry IV, ed. by J.Leszczynski, M.K.Shukla, Springer, New York (2016), p.151.

32. L.N.Kantorovich, J.Phys. C, 21, 5041 (1988).

33. C.Pisani, R.Orlando, R.Nada, in; Cluster Models for Surface and Bulk Phenomena, ed. by G.Pacchioni, P.S.Bagus, F.Parmigiani, NATO ASI Series B, v.283, Plenum, New-York (1992), p.515.

34. B.Paulus, Phys. Rep., 428, 1 (2006).

35. F.Libisch, C.Huang, E.A.Carter, Acc.Chem. Res., 47, 2768 ( 2014).

36. H.Lin, D.G.Truhlar, Theor.Chem.Acc., 117, 185 (2007). 
37. A.V.Luzanov, Theor.Experim.Chem., 22, 489 (1987).

38. P.D.Dacre, C.J.Watts, G.R.J.Williams, R.McWeeny, Mol.Phys., 30, 1203 (1975).

39. M.M.Mestechkin, Metod Matritsy Plotnosti v Teorii Molekul, Naukova Dumka, Kiev (1977) [in Russian].
40. B.N.Parlett, Symmetric Eigenvalue Problem, Prentice-Hall, Englewood Cliffs, New York (1980).

41. G.H.Golub, C.F.Van Loan, Matrix Computations, Johns Hopkins University Press, Baltimore (1990).

42. D.S.Watkins, The Matrix Eigenvalue Problem: GR and Krylov Subspace Methods, SIAM, Philadelphia (2007). 